\title{
Naproxen Induced Psoriasis: A Case Report
}

\author{
Vageeshwari Devuni \\ Pharm.D, CMR College of Pharmacy Kandlakoya, Medchal Road, Hyderabad, Telangana, India
}

\begin{abstract}
How to cite this paper: Vageeshwari Devuni "Naproxen Induced Psoriasis: A Case Report" Published in International Journal of Trend in Scientific Research and Development (ijtsrd), ISSN: 24566470, Volume-3 | Issue-4, June 2019, pp.1133-1135, URL: https://www.ijtsrd.c om/papers/ijtsrd24 004.pdf

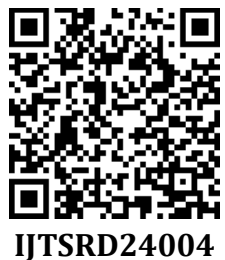

Copyright (C) 2019 by author(s) and International Journal of Trend in Scientific Research and Development Journal. This is an Open Access article distributed under the terms of the Creative Commons

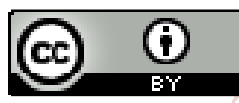
Attribution License (CC BY 4.0) (http://creativecommons.org/licenses/ by $/ 4.0$ )

\section{INTRODUCTION}

Psoriasis is a chronic inflammatory skin disorder clinically. By erythematous sharply demarcated papules and rounded plaques covered by silvery micaceous scale. While the exact causes of psoriasis have yet to be discovered, the immune system and genetics are known to play major roles in its development. Many external factors including infections, stress and medications may exacerbate psoriasis. Some of the most common medications known to trigger or worsen existing psoriasis include NSAIDS, lithium, gold salts, beta blockers and antimalarials(1). Psoriasis is a commonly encountered dermatosis with a variety of internal and external paradoxical factors contributing to the clinical course of the disease. There are several drugs that have been associated with the initiation, exacerbation, and aggravation of psoriasis. Understanding the pathophysiology can provide clues to treatment and management of drug-induced and drug-aggravated psoriasis, which may be indistinguishable from idiopathic. The clinical manifestations of drugassociated psoriasis can range from Plaque type psoriasis to severe Erythroderma, thus warranting astute and sustained clinical observation(2). Psoriasis is a chronic, immunemediated, inflammatory condition seen frequently in the clinical practice with a reported prevalence of 0.6 to 4.8 percent in the general population(3). Some factors known to trigger psoriasis include smoking, alcohol consumption, body mass index(BMI), trauma, infections, endocrine disorders, drugs, and acute withdrawal of systemic or potent topical corticosteroids(4). Drugs have several ways in which they can affect the diathesis of psoriasis including: 1) precipitation of psoriasis de novo in predisposed and non predisposed individuals, 2) exacerbation of pre-existing psoriatic lesions, 3 ) Induction of lesions in clinically normal skin in patients with psoriasis; and 4) Development of treatment resistant psoriasis. The clinical presentation of drug-provoked psoriasis spans the spectrum of generalized plaque psoriasis, palmollantar pustulosis, and Erythroderma. The nails and scalp can also be involved, thus making the distinction of drug associated psoriasis a clinically difficult diagnosis. Drugs that appear to have a strong casual relationship to psoriasis are beta-blockers, lithium, synthetic antimalarials , nonsteroidal anti-inflammatory drugs (NSAIDS), and tetracyclines (5).

\section{CASE:}

A 61yrs old male subject was a known case of Chronic Plaque Psoriasis for which he was using Tab.Methotrexate $7.5 \mathrm{mg}$ since 2.5 yrs. Patient got admitted in Dermatology ward with complaints of red, raised scaly lesions all over the body since 1 month. History of intense itching associated with lesions, scaling over scalp, scaling, fissures, cracks over palms and soles. On general examination patient was conscious and coherent, PR was 80/min, BP-150/100mmhg, Heart/LungsNAD. Cutaneous examination shows multiple erythematous scaly papules and plaques with margins of varying shapes and sizes were present over right and left forearms, hands, legs, foot and back. Auzpitz sign-ve, on scalp and hair diffuse scaling was + , palms and soles were scaly and cracks, fissures, erythema was present, nails- onycholysis+, conductive, oral cavity and genitals were found to be normal. Laboratory findings show complete blood picture: wbc- 
12000cells/mm3, Neutrphils-77.3\%, Monocytes-6.9\%, Hematocrit-36.3\%. Patient used Naproxen an NSAIDS for He adache and neck pain 1 month back for which he developed lesions all over the body. Based on the subjective and objective data it is confirmed as Naproxen induced Psoriasis.
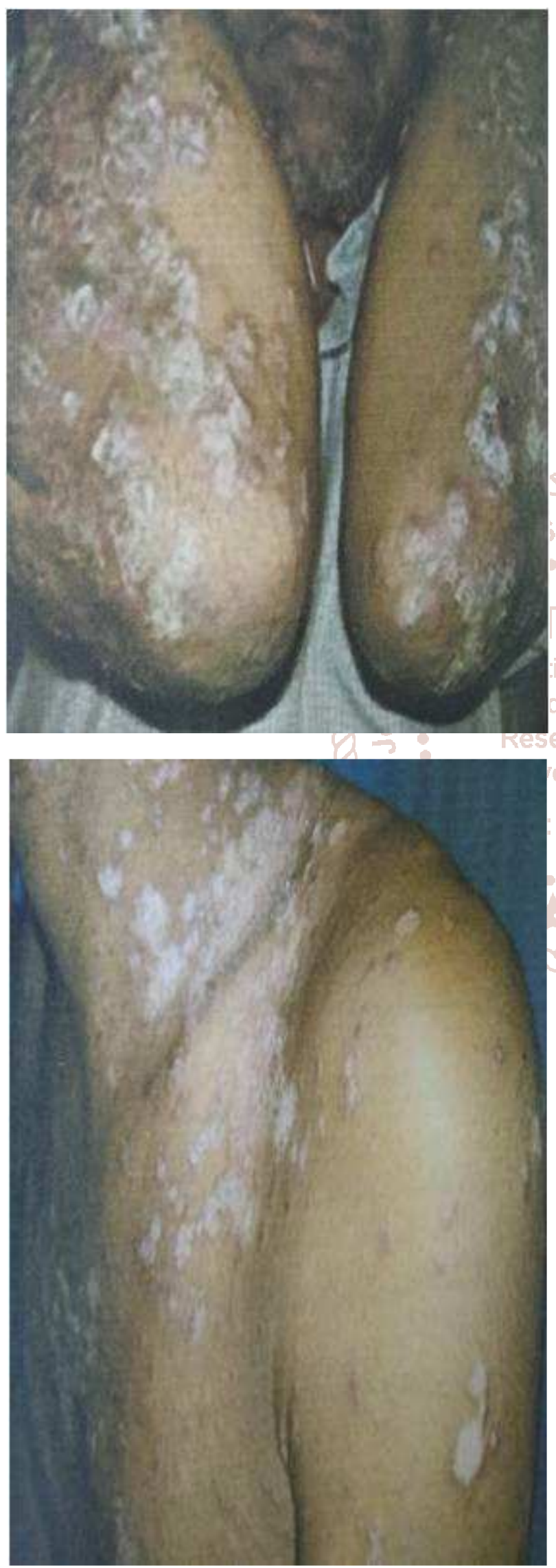

\section{DISCUSSION:}

The patient was a known case of Chronic Plaque Psoriasis and he was using Tab.Methotrexate $7.5 \mathrm{mg}$ for 3 days since 2.5 years. Patient used NSAIDS since 1 month for headache and neck pain. Then he developed lesions all over the body. Drug provoked psoriasis can be divided into two categories. The first category, drug-induced psoriasis, is where discontinuation of the causative drug stops the further progression of the disease. The second category is drugaggravated psoriasis, where the disease progression even after the discontinuation of the offending drug. True druginduced psoriasis tends to occur in a de-novo fashion in patients with no family or previous history of psoriasis. The clinical presentation of these lesions may often mimic the pustular variant of psoriasis, often with no nail involvement or associated arthritis(6). NSAIDS are a class of medications used for treatment of pain and arthritides. NSAIDS are frequently used by patients who have Psoriasis as well as psoriatic arthritis. NSAIDS are available by prescription and over the counter. NSAIDS inhibit the metabolism of arachidonic acid by the cyclo-Oxygenase(COX) pathway leading to accumulation of leukotrienes, which has been postulated to aggravate psoriasis of the NSAIDS, Naproxen was the most common culprit(7). As the patient was a known case of Chronic Plaque Psoriasis and he used NSAIDS which increased the psoriatic lesions. It is important for clinicians to recognize NSAIDS as potential exacerbators of psoriasis. Nevertheless, in some patients, exacerbation of psoriasis and arthritis may coincidently occur simultaneously with the use of NSAIDS. Drug ingestion may result in exacerbation of pre-existing Psoriasis, in induction of psoriatic lesions on clinically uninvolved skin in patients with psoriasis, or in precipitation of the disease in persons without family history of psoriasis or in predisposed individuals(8). The clinical context must be taken into consideration, including effects of concomitant antipsoriatic therapy, and the possible role of other triggering factors, such as infection(9). There have been adverse side effects of NSAIDS reported in patients with psoriasis, particularly with propionic acid derivatives (10). In patients with psoriasis it's responsibility of health care takers to identify any adverse drug reactions when NSAIDS are prescribed along with the regular medication. With an never-ending list of medications, investigators have suggested using the adverse drug reactions probability scale in assessing the relationship between drugs and adverse reactions if a clinicians is faced with a difficult decision in high-risk psoriasis patients on multidrug regimen(11). Drugs with considerable data in the induction/exacerbation of psoriasis include interferons, terbinafine and Benzodiazepines(12).

\section{CONCLUSION:}

Several drugs have been associated with drug provoked psoriasis. Understanding the pathogenesis of drug provoked psoriasis not only helps to achieve a greater appreciation of the disease process, but is also useful in providing guidance for treatment methodologies. In certain cases of drug provoked psoriasis, lesions may become resistant to treatment and hence early recognition and management can help to avoid the issues of nonadherence. Drugs that are considered to have a strong potential risk factors for psoriasis development should be avoided after weighing the risk and benefits of the agent. 


\section{REFERENCES:}

[1] Visnja Milavec-puretic, Marko Lance, Romans Eeovic, Kada Lipozencic. Drug induced Psoriasis; Acta Dermatovenerologica Croatia 19(1),0-0,2011.

[2] Grace K.Kim, DO and James Q. Del Rossi, DO. Drugprovoked Psoriasis: Is it Drug Induced or Drug Aggravated? J Clin Aesthetic Dermatological. 2010 Jan; 3(1): 32-38.

[3] Raychaudhuri SP, Farber E. The prevalence of psoriasis in the world. J EUR Academic Dermatological Venereol.2001;15:16-17.

[4] Jaldi L. Epidemiology of Psoriasis. Cute Drug Targets Inflammatory allergy.2004;3:121-128.

[5] Tsankov N, Irena A, Kasandjieva J. Drug induced Psoriasis: recognition and management. Am J Clin Dermatological.2006;5:426-433.

[6] O'Brian M, Koo J. The mechanism of lithium and betablocker agents in inducing and exacerbating Psoriasis. J Drugs Dermatological. 2006;5:426-433.
[7] Lionel F, Baker B. Triggering Psoriasis: The role of infections and medications . Clin Dermatological. 2007;25:606-615.

[8] Kabbur Hanumanthappa Basavaraj, Navy Mysore Ashok, Ramesh Rashmi et al. The role of drugs in the induction and/or exacerbation of psoriasis. International journal of Dermatology 49(12), 1351$1361,2010$.

[9] EA Abel.Diagnosis of drug-induced psoriasis. Seminars in Dermatology 11(4),269-274,1992.

[10] Cohen AD, Bonneh DY, Reuveni M,et al.Drug exposure and psoriasis vulgaris:case-control and case-crossover studies. Acta Derm Venereol.2005;85:299-303.

[11] Naranjo CA, Busto U, Seller EM, et al. A Method for estimating the probability of adverse drug reactions. Clin Pharmacol Ther.1981;30:239-245.

[12] Katz M, Seidenbaum M, Weinrauch L. Penicillin induced generalized pustular psoriasis. J Am Academic Dermatol.1987; 17: 918-920.

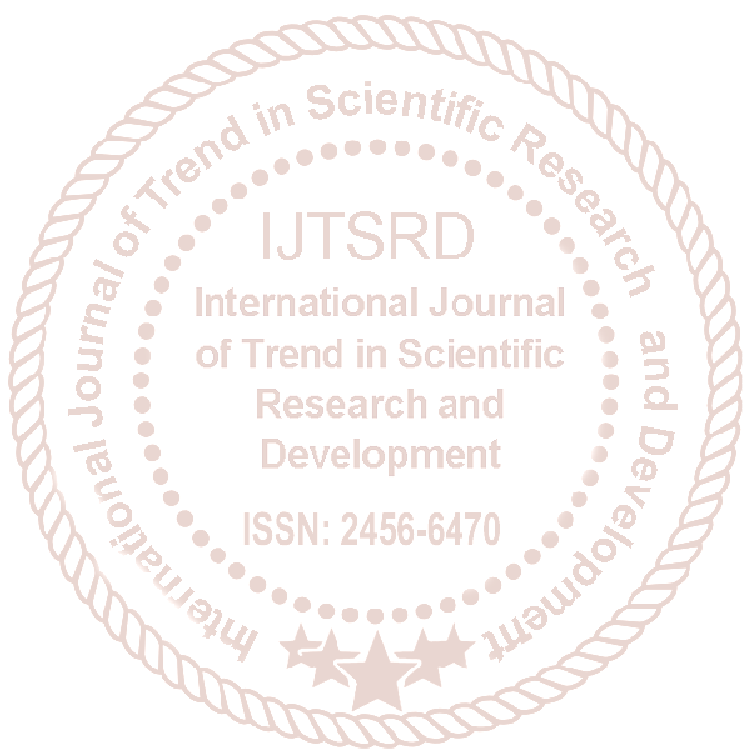

